\title{
LA GLOBALIZACIÓN Y LA ENSEÑANZA DE LA PSICOLOGÍA EN ARGENTINA
}

\author{
Hugo Klappenbach ${ }^{*}$
}

\begin{abstract}
RESUMEN. El trabajo aborda tres cuestiones. En primer lugar, analiza brevemente a la historia de la enseñanza de la psicología en Argentina, subrayando la importancia adquirida por el psicoanálisis en la tradición universitaria psicológica. En segundo lugar, desarrolla algunos de los estudios y algunas de las líneas de debate contemporáneo sobre formación universitaria del psicólogo, comenzando por algunos estudios clásicos en los Estados Unidos y concluyendo en la propuesta de diplomatura en psicología, actualmente en debate en los países miembros de la Unión Europea. En tercer lugar, analiza el impacto generado por la globalización en los procesos de formación del psicólogo en Argentina, especialmente a partir del impacto del llamado Mercosur educativo. En ese marco, se analiza críticamente la situación de los programas de psicología en las universidades argentinas.
\end{abstract}

Palavras-chave: enseñanza, programas, psicología.

\section{GLOBALIZATION AND EDUCATION IN PSYCHOLOGY IN ARGENTINA}

\begin{abstract}
This paper approaches three subjects. Firstly, it briefly analyzes the history of psychology undergraduate programs of in Argentina, in which psychoanalytical bias is emphasized. Secondly, it reviews some important studies and reports concerning undergraduate programs and professional training in Psychology, beginning with some classical reports in USA, finishing with the proposal of an European Diploma in Psychology, which is now under evaluation in European countries. Thirdly, globalization effects, specially those from educational Mercosur, on teaching of psychology at undergradute programs and professional training in psychology in Argentina are analyzed. In such context, a critical report on psychology undergraduate programs in the country is reviewed.
\end{abstract}

Key words: education, programs, psychology.

\section{LA ENSEÑANZA DE LA PSICOLOGÍA EN ARGENTINA}

La primera cátedra de Psicología en Argentina tuvo lugar en la Facultad de Derecho y Ciencias Sociales a cargo del abogado Ernesto Weigel Muñoz. Un año después, en la Facultad de Filosofía y Letras, se organizaba una segunda cátedra de psicología, que primero estuvo a cargo de Rodolfo Rivarola y desde 1901 de Horacio Piñero (Klappenbach, 1987).

Sin embargo, los primeras proyectos de formación en psicología se producirían a partir de la segunda mitad del siglo, tal como venía ocurriendo en Europa y en la mayoría de los países latinoamericanos, aunque no serían en Psicología sino en Psicotecnia y Orientación Profesional: la carrera de Psicotécnico y Orientador Profesional, que la Universidad Nacional de Tucumán organizó en 1950 (Rossi, 1997); la Especialización en Psicología que se organizó en la Universidad Nacional de Cuyo en 1953, bajo la dirección de Plácido Horas (Klappenbach, 1995); y la Carrera de Asistente en Psicotécnica, que en el mismo año planificó en Rosario la Universidad del Litoral (Ascolani, 1988; Gentile, 1989).

* Licenciado en Psicología y Doctor en Historia.Profesor Titular Efectivo de Historia de la Psicología de la Universidad Nacional de San Luis. - Investigador del Consejo Nacional de Investigaciones Científicas y Técnicas (CONICET). Docente-Investigador Categoria 1 del Programa de Incentivos a la Investigación. Consejo Interuniversitario Nacional - Secretaría de Políticas Universitarias de la Nación. Primer Coordinador de la "Task Force on History of Psychology" de la Interamerican Society Psychology (SIP). - Liaison en Argentina de la Division 52 (International Psychology) de la American Association (APA). Editor de los Cuadernos Argentinos de Historia de la Psicologia. 
El desarrollo de aquellos proyectos, salvo en la Universidad Nacional de Tucumán, fueron irregulares, y se subsumirían en las futuras carreras de psicología, que se organizarían entre 1954 y 1959, como consecuencia de una recomendación realizada en el Primer Congreso Argentino de Psicología, reunido en Tucumán en 1954. Aun cuando luego de 1955 el clima político se modificaría substancialmente, todavía bajo la invocación de aquel congreso, entre 1954 y 1959, comenzaron a organizarse carreras universitarias de psicología; primero, en universidades nacionales, en Rosario, Buenos Aires, San Luis, Tucumán, Córdoba y La Plata (Klappenbach, 2000a) y en seguida en universidades provinciales y privadas (tabla 1).

Como consecuencia de la organización de aquellas carreras universitarias, apenas iniciada la década de 1960 surgieron los graduados en psicología en el país, poniendo a fin a la etapa de la llamada "psicología sin psicólogos" (Vilanova \& Di Doménico, 1999). Desde entonces, se consolidaría el perfil de la psicología como profesión, colocando en el centro de la escena, el debate en torno al rol del psicólogo.

Se ha analizado que, en uno de los extremos del debate, se sostenía que el psicólogo era simplemente un psicoanalista (Harari, 1970, Klappenbach, 2000b). Desde esa posición, se consideraba que el psicoanálisis, tal como lo había planteado Freud, debía considerarse al mismo tiempo una teoría, una terapéutica y un método de investigación. Entonces, desde un punto de vista estrictamente freudiano, el psicoanálisis era nada más que una psicología, ni siquiera una psicología médica, sino una psicología $a$ secas, cuyo objeto de estudio era el inconsciente.

En el otro extremo del debate, se sostenía que "el psicólogo es - aun por sola presencia- un agente de cambio" (Bleger, 1966, p. 75, el subrayado añadido). Desde esa posición, se señalaba la necesidad de diferenciar entre el rol del psicólogo y aquél del psicoanalista, proponiendo para el psicólogo el campo de la higiene mental, de la profilaxis (Danis, 1969; Klappenbach, 2000b).

Al mismo tiempo, diferentes posiciones se movían entre uno y otro extremo del debate, como así también diversos matices e inflexiones de ambas posturas. De todas manera, la contradicción entre el rol del psicoanalista y el de agente de cambio era relativa. Los que proponían el modelo del agente de cambio se inspiraban en las enseñanzas de José Bleger, un prominente didácta de la Asociación Psicoanalítica Argentina (APA) y en ese sentido se señalaba que el psicólogo podría disponer de conocimientos psicoanalíticos o inclusive que "que no puede formarse un psicólogo en forma científica, si carece de preparación en Psicoanálisis. Creo que esto es absolutamente indispensable" (Danis e col., 1970, p. 118).

Tabla 1. Carreras de Psicología en Argentina, en los primeros diez años (1954-1964).

\begin{tabular}{lll}
\hline \multicolumn{1}{c}{ Fecha de Creación } & \multicolumn{1}{c}{ Ciudad } & \\
\hline 1954 (Mayo) & Rosario & Universidad Nacional del Litoral \\
1957 (Marzo) & Buenos Aires & Universidad de Buenos Aires (nacional) \\
1958 (Febrero) & San Luis & Universidad Nacional de San Luis \\
1958 (Noviembre) & La Plata & Universidad Nacional de La Plata \\
1958 (Diciembre) & Córdoba & Universidad Nacional de Córdoba \\
1959 (Agosto) & Tucumán & Universidad Nacional Tucumán \\
1959 (Agosto) & Buenos Aires & Universidad del Salvador (privada). En 1955 comenzó como un Instituto de Psicología. \\
1959 (Agosto) & Córdoba & Universidad Católica de Córdoba (privada) \\
1960 (Mayo) & Mar del Plata & Universidad de Mar del Plata (provincial). En 1960 comenzó como Programa del Instituto de Ciencias de la Educación. In 1966 \\
& & sería incorporado a la Universidad Provincial de Mar del Plata. Fue cerrado en 1976 durante la dictadura militar y reabierta en \\
1961 (Marzo) & Buenos Aires & Facultad Libre de Psicología (privada). Anexada a la Universidad Católica Argentina. Había comenzado en 1960 como Instituto. \\
1962 (Marzo) & Buenos Aires & Universidad del Museo Social Argentino (privada) \\
1963 (Marzo) & Tucumán & Universidad del Norte Santo Tomás de Aquino (privada) \\
1963 (Agosto) & Mendoza & Facultad de Antropología Escolar (provincial). Administración General de Escuelas de la Provincia de Mendoza. Fue clausurada \\
& & definitivamente en 1997. \\
1964 (Marzo) & Buenos Aires & Universidad Argentina John F. Kennedy (privada). \\
\hline
\end{tabular}

Fuentes: Para las universidades nacionales: Klappenbach, 2000a, Alderete, 1999, comunicación personal; Ascolani, 1988, Barrionuevo \& García Marcos, 1973; Gentile, 1989; Monasterio, 1965; Llapur, 1972; Torninbeni, 1999; Universidad de Buenos Aires, 1957; Universidad Nacional de Cuyo, 1958. Para la Universidad Provincial de Mar del Plata: Diez, 1994.

Para la Facultad de Antropología Escolar de la Provincia de Mendoza: Guisasola, 2003, comunicación personal. Para las universidades privadas: Ministerio de Educación, Ciencia y Tecnología. Secretaría de Políticas Universitarias, 2002 y gentileza de las universidades.

A tono con ese debate desde finales de la década del cincuenta, los contenidos psicoanalíticos irían permeando las carreras de psicología en casi todo el país, aun en las universidades católicas. Es difícil encontrar una sola razón que explique ese fenómeno. Pero no cabe duda que los primeros psicoanalistas que 
comenzaron a enseñar tanto en la Universidad de Buenos como en la del Litoral, sobre todo Bleger, partían de la concepción de que la psicología constituía un "oficio" (Bleger, 1962). En ese sentido, si la psicología no constituía una ciencia sino un oficio, sus conocimientos científicos debían provenir de otro lugar, en este caso del psicoanálisis.

El sesgo psicoanalítico, entonces, impregnó toda la formación del psicólogo entre 1960 y el fin de la dictadura militar en 1983. Aun en las últimas dos décadas ese sesgo no se ha modificado totalmente. En efecto, en la Universidad Nacional de Rosario se ha reconocido una marcada hegemonía psicoanalítica lacaniana que también puede observarse, aunque menos pronunciada cuantitativamente, en las universidades nacionales de Buenos Aires, Córdoba y Tucumán y en muchas universidades privadas. Sin embargo, desde la recuperación democrática se ha diversificado el perfil de formación universitario, tanto en relación con las macro-teorías dominantes, como en relación con los campos de la práctica.

\section{FORMACIÓN UNIVERSITARIA DEL PSICÓLOGO. PANORAMA INTERNACIONAL.}

Desde la década de 1990 se han incrementado notablemente los estudios relacionados con la formación universitaria del psicólogo. Una de las líneas destacadas de estos estudios se ha centrado en el análisis y definición de las características de las competencias y capacidades que reúnen los psicólogos tras el entrenamiento universitario (Bartram, 1996; Blanco, 1998; Hayes, 1996; Klappenbach, 2000a; Mc Govern, Furumoto, Halpern, Kimble \& Mc Keachie, 1991; Peterson, Peterson Abrams, \& Stricker, 1997; Poortinga \& Lunt, 1997). Una dirección coincidente de los estudios recientes, ha priorizado el análisis de estándares internacionales en la formación profesional del psicólogo (Lunt, 1999; Nixon, 1990; Poortinga \& Lunt, 1997). En una dirección divergente, tampoco han faltado los estudios centrados en la imposibilidad de hallar cualquier núcleo común en la formación de psicólogos, toda vez que la psicología se caracteriza por su diversidad (Benjamin, 2001; Griggs, Proctor \& Bujak-Johnson, 2002; Lipsey, 1990).

Sin embargo, la cuestión generaría algunos estudios e informes institucionales clásicos entre principios de siglo y el fin de la Segunda Guerra Mundial (Routh, 2000).

En 1933, un Comité de la American Psychological Association (APA) encabezado por Walter Hunter, dirigió una encuesta a 22 programas de doctorado en psicología en Estados Unidos. Dicho
Comité, por una parte, reconoció que salvo por un par de excepciones, todos los programas coincidían en al menos tres áreas: psicología experimental, estadística y teoría-historia de la psicología. Por la otra, no obstante ese reconocimiento, el Comité no realizó ningún tipo de recomendación orientado a un entrenamiento estandarizado o que contemplara un núcleo de formación común (core curriculum) (Benjamin, 2001).

En 1947 el entonces presidente de la Universidad de Harvard, James Conant, convocó a doce personalidades notables, la conocida Comission of Twelve, conformada por seis personas que provenían del campo de la psicología y seis ajenos a dicho campo, a estudiar el impacto de la psicología en la formación universitaria de médicos, sociólogos, maestros, ingenieros y hombres de negocios y administración (Vilanova, 1996). La Comisión recomendaría centrar el estudio de la psicología en cursos relacionados con matemática, ciencias naturales y de la vida y ciencias sociales; en esa dirección, consideraría a la psicología como un vínculo entre las ciencias sociales y las ciencias biológicas (McGovern, 1992).

Dos años después, la Administración de Veteranos de Guerra, con el respaldo del Servicio de Salud Pública de los Estados Unidos, preocupada por las perturbaciones mentales de un gran número de excombatientes, convocó a la Asociación de Psicólogos Americanos a una reunión destinada a discutir la manera por la cual el psicólogo podía ser de utilidad en los problemas de los veteranos de guerra, lo cual daría lugar a la célebre Conferencia de Boulder, Colorado (Raimy, 1950).

No era el primer intento por considerar la formación de los psicólogos en el campo de la clínica. En plena guerra, un Comité convocado por el Instituto Psiquiátrico de Nueva York, había recomendado una formación doctoral en base a cuatro años, en el cual, en el primero, se procuraría una formación en medicina y psicología, con fuerte acento en el estudio de la psicopatología, en el segundo año se profundizaría en psicometría y terapia, el tercer año estaría destinado a un internado y el último a la preparación de la tesis (Shakow, 1942)

Asimismo, en 1947, la Asociación de Psicólogos Americanos había constituido el Comité de Entrenamiento en Psicología Clínica, también presidido por Shakow, cuyo informe de 1947, fue la base inicial de discusión en la Conferencia de Boulder (Baker \& Benjamin, 2000).

En ese sentido, al reunirse la Conferencia sobre Entrenamiento en Psicología Clínica en Boulder, Colorado, el problema sobre la formación en 
psicología clínica estaba suficientemente discutido. En Boulder se diseñó un modelo relativamente estandarizado sobre formación universitaria en psicología, que sería conocido como el modelo científico-profesional (scientist-practitioner model). Dicho modelo, abarcaba cuatro años de formación durante los cuales se combinaba la formación académica y la aplicada, y se enfatizaba un año de internado y posteriormente el $\mathrm{Ph}$ D. (Baker \& Benjamin, 2000; Belar, 1992a, 2000). Al mismo tiempo, se especificaban los contenidos que debía incluir la formación del psicólogo:

La educación de grado debía incluir psicología general (sistemática, experimental, psicología social), psicología clínica (teoría, método, técnica), trabajo de campo (variedad de problemas y niveles de responsabilidad) e investigación. La investigación era enfatizada como una base para la comprensión del comportamiento humano, para el mejoramiento de las técnicas diagnósticas, para el desarrollo de métodos de tratamiento y finalmente dirigidos a la prevención de la higiene mental. Doce áreas del núcleo central clínico fueron propuestas: fisiología humana, teoría de la personalidad, psicología del desarrollo (determinantes biológicos, sociales y culturales); relaciones sociales (psicología social, sociología, economía, antropología); psicopatología; medicina clínica y psiquiatría clínica (modelo médico); psicoterapia y procedimientos de alivio (remedial procedures); metodología de la investigación clínica; relaciones profesionales e interprofesionales; recursos y organizaciones comunitarias; experiencia de practicum e internado. (Dana \& May, 1987, p. 8).

Las recomendaciones de Boulder no podían ocultar las preocupaciones clínicas que habían convocado la Conferencia. Inclusive, se proponía "un amplio desarrollo de la psicología clínica a lo largo de la líneas que se extienden desde el francamente psicótico o enfermo mental hasta la clientela relativamente normal que necesita información, asesoramiento vocacional y alivio" (Raimy, 1950, p. 113, la traducción es mía). Sin embargo, su énfasis en la psicología, tanto como una ciencia como una profesión, haría posible la extensión del modelo a otras áreas de aplicación de la psicología, aun cuando se haya señalado que su mayor falencia radica en su aceptación acrítica del modelo y lenguaje médico y psiquiátrico (Albee, 2000)

Desde aquellos estudios clásicos, han sido constantes los análisis y estudios relacionados con la formación y entrenamiento en psicología en los Estados Unidos, tanto los estudios empíricos sobre la currícula de grado como los informes producidos por comité de expertos, task forces o conferencias (McGovern, 1992). Entre las últimas, hay que destacar aquellas conferencias que se centraron en la formación a nivel de los programas de grado (undergraduate level), aquellas centradas en el entrenamiento profesional de psicólogos que siguieron a Boulder y aquellas centradas en la formación postdoctoral y el internado: Cornell, 1951; Standord, 1955; Miami Beach, 1958; Michigan, 1960; Princeton, New Jersey, 1961; Chicago, 1965; Vail, Colorado, 1973; Arden House, New York, 1983; Hilton Head Island, South Carolina, 1985; Mission Bay, California, 1986; Utah, 1987; Gainesville, Florida, 1987; Gainesville, Florida, 1990; Maryland, 1991 (Belar, 1992a; Belar, 1992b; Belar \& Perry, 1992; Ellis, 1992; Fox \& Barclay, 1989; Lloyd \& Brewer, 1992 ).

En tales encuentros, se afianzaría el modelo científico-profesional originado en Boulder, pero al mismo tiempo se diversificaría. Así, en las recomendaciones finales de la Conferencia de Miami de 1958 , se destacaría la necesidad de una formación que abarcara la mayor variedad de campos del ejercicio profesional de los psicólogos, y no solamente el clínico. Asimismo, se señaló la importancia de la regulación de la profesión a través de la acreditación de los programas de formación y los criterios para el otorgamiento de matrículas para el ejercicio profesional (Belar, 1992a; Sheridan, Matarazzo \& Nelson, 1995).

Otra cuestión de relevancia en la Conferencia de Miami, estuvo dada por la imposibilidad de definir un núcleo en la formación en psicología, no obstante que los participantes acordaron en reconocer que ese núcleo efectivamente existe:

\footnotetext{
Dos interesantes conclusiones aparecieron (...) Primero, que existe un núcleo común (common core). Segundo, que nosotros no podríamos especificar en que consiste, cuestion de no desalentar la innovación imaginativa en el entrenamiento de grado (Roe e col., 1959, p. 44, citado por Benjamin, 2001, p. 738) (la traducion es mia).
}

Por su parte, la Conferencia celebrada en 1991 en el St Mary's College, Maryland ensanchó notablemente el rango de cuestiones tratadas. En particular destacó que:

1. El objetivo general (general goal) de la formación del psicólogo consiste en "enseñar a los estudiantes a pensar como científicos sobre la conducta y la 
experiencia" (Lloyd \& Brewer, 1992, p. 275, la traducción es mía).

2. Asimismo, se identificaron seis objetivos específicos del curriculum: a) atender a la diversidad humana; b) amplitud y profundidad del conocimiento; c) competencia metodológica; d) experiencia práctica y aplicada; e) adquisición de habilidades comunicativas; f) sensibilidad y preocupación por las cuestiones éticas (Blanco, s/f; Lloyd \& Brewer, 1992).

3. En relación con la secuenciación y correlatividad de los estudios, la Conferencia de St. Mary planteó cuatro tipo de cursos: introductorios, metodológicos, cursos de contenidos y experiencias integradas (integrative or capstone experiences).

4. Desde el punto de vista de las prácticas docentes, se enfatizó el papel de estrategias activas de aprendizaje: simulaciones, demostraciones, trabajo interactivo, juegos, resolución de problemas, etc.

5. También se recomendó la necesidad de establecer sistemas de cooperación entre los distintos departamentos de psicología de las universidades (Blanco, s/f; Lloyd \& Brewer, 1992).

Es de destacar que la Conferencia de Maryland enfatizó la importancia de que la psicología mantuviera su relación con otras disciplinas sociales y significó el informe institucional más abarcativo sobre los distintos tópicos relacionados con la formación del psicólogo.

En Europa, por su parte, la preocupación por la formación del psicólogo sería central en la European Federation for Professional Psychological Associations (EFPPA), la cual desde la Asamblea General de Londres de 2001, modificó su denominación por el de European Federation of Psychological Associations (EFPA), nombre que reflejaba la necesidad de abarcar tanto el campo profesional como el científico de la psicología.

En aquel continente, las carreras de psicología surgieron por lo general con posterioridad a la Segunda Guerra Mundial, igual que en América Latina. Dos estudios de mediados de la década pasada, revelaban que en Europa coexistían por lo menos tres modelos distintos de currícula: a) una currícula de cinco años de duración generalista; b) una currícula de cinco años de duración, enfatizando una especialización en el área educacional o clínica u otra; c) una currícula de seis o siete años, donde los tres o cuatro primeros se limitan a un visión general, al cabo de los cuales, pero a nivel de postgrado, se centraba el futuro psicólogo en una sola especialización (Lunt, 1994; Newstead, 1994).

No obstante, el panorama de la psicología europea evidencia dos tendencias contradictorias. Por una parte, una tendencia centrada en las diferencias entre los distintos países, tanto en lo relacionado con la formación universitaria del psicólogo como con los aspectos legales que regulan los títulos y la profesión (Lunt, 1999; Pulverich, 1998; Wilpert, 1999).

Por la otra, una tendencia a la homogeneización de la enseñanza y de otros aspectos regulables de la disciplina, que se ha incrementado notablemente en la última década (Lunt, 1998, 2002; Peiró \& Lunt, 2002; Poortinga \& Lunt, 1997; Wilpert, 2002).

En relación con esta última tendencia, hay que destacar que la formación del psicólogo en los países de la Unión Europea está enmarcada en la directiva 48/89 de la entonces Comunidad Económica Europea, la cual promovía la libre circulación de profesionales en el camino de la unificación europea. El artículo $3^{\circ}$ de dicha directiva establecía lo siguiente:

Cuando, en el Estado miembro de acogida, el acceso o el ejercicio de una profesión regulada estén sujetos a la posesión de un título, la autoridad competente, basándose en el argumento de insuficiencia en la cualificación, no podrá denegar a un nacional de otro Estado miembro el acceso a tal profesión o su ejercicio en las mismas condiciones que a los ciudadanos de su propio país:

a) si el solicitante posee el título requerido por otro Estado miembro para acceder a dicha profesión o ejercerla en su territorio, y ha sido obtenido en un Estado miembro, o

b) si el solicitante ha ejercido dicha profesión a tiempo completo a lo largo de dos años durante los diez años anteriores en otro Estado miembro el cual no regula dicha profesión (...) (The Council of The European Communities, 1989, p. 19; la traducción es nuestra).

Según una investigación encarada conjuntamente en varios países miembros de la Unión Europea y subsidiada por el Programa Leonardo Da Vinci, la Directiva 48/89 todavía no se estaría cumpliendo plenamente (Project EuroPsyT, 2001).

No obstante, esa directiva y más ampliamente el proceso de unificación europea, condujo, ya en 1992, a que la EFPA aprobara unos estándares mínimos en la formación del psicólogo, necesarias para el ejercicio de la profesión en cualquier país de la comunidad 
(European Federation of Professional Pschologists Association, 1992).

Más recientemente todavía, en marzo del 2002 el Presidente de la EFPA, Tuomo Tikkanen, reconocía que una nueva Directiva de la Unión Europea de febrero de 2002 posibilitaba la implantación de un Diploma Europeo común para todos los países miembros de la Unión, sobre la cual viene trabajando el Grupo de Trabajo en Enseñanza de la Psicología de la EFPA y el proyecto de investigación financiado por el Programa Leonardo Da Vinci (Tikkanen, 2002b; Wilpert, 2002). Los criterios para el establecimiento de tal diplomatura común partirían de la base de:

un título de grado, un título de Master o un nivel equivalente de entrenamiento universitario y por lo menos un año de práctica supervisada, lo que sumaría seis años (Tikkanen, 2002a, p. 162) (la traducción es nuestra).

En la última Asamblea de la EFPA, llevada a cabo en Viena en julio del 2003, se resolvió que en mayo del 2004, dicha institución presentaría ante la Comisión Europea su propuesta de una diplomatura única para los países de la Unión Europea (European Federation of Professional Pschologists Association, 2003; Tikkanen, 2003).

En cuanto a América Latina, merecen destacarse los estudios de Amalio Blanco realizados en el marco de la Organización de Estados Americanos y los trabajos sobre la situación en el Mercosur (Blanco, 1998; s/f; Blanco, Dembo, Di Doménico, Pineda, \& Rojo, 1993).

Asimismo, otra referencia clásica está dada por la Conferencia reunida en Bogotá en 1974, que diseñó lo que comenzaría a conocerse como el "modelo latinoamericano" de entrenamiento en psicología. El mismo insistía en la necesidad de una currícula "generalista", que tuviera en cuenta la formación básica capaz de fundamentar el desempeño en los distintos campos de aplicación, y reconocía a la psicología tanto su aspecto de ciencia como de profesión (Ardila, 1975, 1978).

La Conferencia comenzaba por señalar algunos déficits en la formación en psicología en la región, de los cuales me interesaría subrayar al menos dos:

$1^{\circ}$ - déficit de personal entrenado en las áreas de educación, salud mental, investigación sobre el comportamiento;

$2^{\mathrm{a}}$ - el rol profesional del psicólogo se confunde con el del psiquiatra, el psicoanalista o el pedagogo;

Frente a tal situación, la Conferencia proponía:
- Reconocimiento del doble carácter de la psicología: ciencia y profesión.

- Necesidad de un entrenamiento de los psicólogos según pautas similares en todos los países de la región.

- Entrenamiento en todas las áreas de intervención profesional.

- Perfeccionamiento en los últimos semestres en alguna de dichas áreas.

- Necesidad de un título generalista que permitiera desempeñarse en las diferentes áreas.

- Obtención del titulo luego de dos requisitos: escritura de una tesis y entrenamiento práctico (practicum o pasantía) en un área de intervención.

\section{IMPACTO DE LA GLOBALIZACIÓN EN LA FORMACIÓN DEL PSICÓLOGO EN ARGENTINA}

Ante todo, quisiera comenzar señalando que la cuestión de la globalización merece diferentes lecturas y parece ser un fenómeno contradictorio. Porque, por una parte, resulta incuestionable el pronunciado deterioro en términos de justicia social y de bienestar general para la mayoría de la humanidad que ha acompañado la globalización entendida como un proceso de transnacionalización y concentración económica.

Pero por la otra, desde Kant hasta Marx, toda la filosofía política crítica, ha planteado la necesidad de superar las limitaciones de los estados nacionales y sus burguesías hegemónicas, a favor de procesos de internacionalización de la esfera pública.

En ese contexto, la cuestión de la enseñanza de la psicología en las universidades argentinas, no puede desconocer su relación con los procesos relacionados con la conformación de un bloque político y económico regional. Es de destacar en esa línea de pensamiento que el 7 de mayo de 2003, se constituyera la Red Iberoamericana para la Acreditación de la Calidad de la Educación Superior (RIACES), por el momento integrada por funcionarios de Argentina, Chile, Colombia, Costa Rica, Cuba, Ecuador, España, México y Venezuela (Red Iberoamericana para la Acreditación de la Calidad de la Educación Superior, 2003).

Es decir, los estudios y debates sobre la formación del psicólogo en Argentina, y sobre todo, sobre la necesidad en establecer estándares mínimos de formación en las 30 carreras que hoy dictan carreras de psicología en el país, se superpone con la necesidad de garantizar la libre circulación de bienes y personas, 
por ejemplo psicólogos, en el Mercosur y países asociados.

En Argentina, los estudios más destacados sobre formación universitaria del psicólogo se han venido realizando desde comienzos de la década de 1990. Desde una perspectiva general, se han ocupado investigadores de la Universidad Nacional de Mar del Plata, Alberto Vilanova y Cristina di Doménico (Di Doménico, 1996, 1999a; Di Doménico \& Vilanova, 1990a, 1990b; Vilanova, 1993, 1994, 1997; Vilanova \& Di Doménico, 1999). Interesados por la formación del psicólogo en su propia universidad, son de destacar los estudios encarados en la Universidad Nacional de La Plata (Piacente, 1994; Piacente, Compagnucci, Schwartz \& Talou, 2000). Telma Piacente, por su parte, también se ha dedicado a investigar el lugar del psicoanálisis en la formación del psicólogo (Piacente, 1998). Esta última problemática también ha sido de mi interés (Klappenbach, 1998) aunque mi mayor preocupación se ha centrado en la formación del psicólogo en todo lo relacionado con las prácticas de investigación (Klappenbach, 2003).

Desde el punto de vista institucional, son de destacar los Informes y propuestas generados por la Asociación de Unidades Académicas en Psicología (AUAPsi). La constitución de AUAPsi surgió de una reunión llevada a cabo junio de 1991 en la ciudad de Córdoba, cuyo tema principal era la "jerarquización de la carrera de Psicología" (Asociación de Unidades Académicas en Psicología, s/f, p. 1). La AUAPsi se organizó formalmente en una reunión posterior llevada a cabo en Rosario el 15 de agosto de 1991, con el objetivo de "promover la interrelación entre las distintas unidades académicas de psicología del país, con el objetivo permanente de mejorar la formación de grado y posgrado, la investigación y la extensión universitaria" (Asociación de Unidades Académicas en Psicología, s/f, p. 8).

A tal fin, el artículo tercero, inciso $a$ de los Estatutos indicaba que una de sus funciones era "propender al logro de objetivos comunes en cuanto al perfil del graduado, su curriculum y grado académico, acorde con las necesidades nacionales y regionales y con los desarrollos actuales de la Psicología en el mundo" (Asociación de Unidades Académicas en Psicología, s/f, p. 8). Por su parte, el inciso $i$ señalaba: "propender a la homogeneidad de los contenidos curriculares y de los títulos y grados académicos otorgados" (Asociación de Unidades Académicas en Psicología, s/f, p. 9).

Es decir que la necesidad de encontrar estándares comunes y homogéneos para la formación del psicólogo estaba entre los objetivos fundantes de la institución y es previo a la constitución del Mercosur educativo. En efecto, el Mercosur fue formalizado a través del Tratado de Asunción de marzo del 1991 (Mercosur, 1991), y el llamado Mercosur educativo surgió de la Primera Reunión de Ministros de Educación de los países miembros en Brasilia el 21 de diciembre de 1991.

En aquella reunión, se acordó un Protocolo de Intenciones para el área educativa, que declaró en su punto quinto, "el propósito de favorecer la articulación, la equiparación y la convalidación de los estudios entre los diferentes Sistemas Educativos, en todos sus niveles y modalidades" (Mercosur. Reunión de Ministros de Educación, 1991, p. 2), y acordó en su punto tercero, la "armonización de los sistemas educativos" (Mercosur. Reunión de Ministros de Educación, 1991, p. 3).

A efectos de alcanzar esa armonización de los sistemas educativos, el Protocolo de Intenciones promovía las siguientes acciones:

3.1. Propiciar un conjunto de medidas que tiendan a superar las barreras jurídicas y administrativas, que permita la movilidad e intercambio de personas y bienes de las áreas científicas, técnicas y culturales;

3.2. Establecer un sistema de información que posibilite conocer los datos educativos relevantes de los Países Partes, así como el acceso al conocimiento disponible sobre mercado de trabajo y sectores de actividad;

3.3. Crear una red institucional de cooperación técnica, preferentemente en las áreas de Educación Inicial Primaria, Media, Especial y de Jóvenes y Adultos;

3.4. Tipificar los perfiles mínimos de formación profesional y técnica, que posibiliten la equivalencia de los estudios y títulos, facilitando el ejercicio profesional en los Países Partes;

3.5. Compatibilizar los perfiles para la formación de los recursos humanos de nivel superior (terciario y universitario), especialmente los contenidos de las disciplinas fundamentales en las áreas e intereses del MERCOSUR, posibilitando el establecimiento de mecanismos que faciliten la circulación de alumnos, docentes y profesionales en la región." (Mercosur. Reunión de Ministros de Educación, 1991, pp. 4-5).

En cualquier caso, aun cuando los objetivos de armonización curricular para las carreras de psicología propuesto por AUAPsi, precediera al Protocolo de 
Intención del Mercosur, resulta indudable que el Mercosur, con todos sus vaivenes y en particular el denominado Mercosur Educativo, ha sido un dinamizador fundamental de todas las reflexiones y modificaciones curriculares en psicología actualmente en curso en Argentina y Uruguay.

De hecho, al mismo tiempo que los países miembros del Mercosur suscribieran el "Protocolo de Admisión de Títulos y Grados Universitarios para el Ejercicio de Actividades Académicas en los Países del Mercosur", el 11 de junio de 1997 (Mercosur, 1997), AUAPsi encaró un Proyecto de Mejoramiento de la Calidad Educativa, que se componía de dos programas:

a) Programa de Capacitación Docente en áreas consideradas de vacancia disciplinar. Estaba destinado a docentes universitarios con responsabilidades significativas en el diseño de programas de las materias, y abarcaba básicamente dos grandes áreas: metodología de la investigación y procesos básicos.

b) Programa de Formación de Especialistas en Innovación Curricular. El objetivo del Programa consistía, a un mismo tiempo en capacitar psicólogos en todo lo relacionado con la temática de enseñanza de la psicología procurando que dicha capacitación no fuera únicamente teórica sino que se fundamentara en la elaboración de un diagnóstico de cada una de las carreras de psicología de universidades nacionales y luego en un diagnóstico que sintetizara la situación de todas las carreras, seguido de una serie de recomendaciones sobre las medidas a adoptar en el futuro y que posibilitaran el mejoramiento de la calidad de los graduados en psicología en Argentina y Uruguay (Asociación de Unidades Académicas -AUAPsi-, 1996; Di Doménico, 1999b).

El Programa de Formación de Especialistas en Innovación Curricular se desarrolló entre 1997 y 1999. En primer lugar, se elaboró un diagnóstico de la situación actual de la enseñanza de la psicología en Argentina y Uruguay en su conjunto y en cada una de las carreras de psicología de las universidades públicas de ambos países (Asociación de Unidades Académicas, 1998). El mismo Programa, concluyó con un Protocolo de Acuerdo que sintetizaban los primeras estándares comunes para la formación del psicólogo en Argentina y Uruguay, apuntando sobre todo a aquella necesidad de encontrar puntos comunes con la formación en los países del Mercosur y Estados asociados (Asociación de Unidades Académicas, 1999).
A los efectos del diagnóstico y las recomendaciones, AUAPsi tomó en consideración los distintos momentos intervinientes en el proceso de diseño, evaluación e implementación efectiva de los planes de estudio, desde un marco conceptual que reconocía tres niveles fundamentales en la Estructura del Diseño Curricular, conocidos como tres niveles de concreción del curriculum (Coll, 1992b). Dicha "matriz de análisis y planificación curricular permite delimitar cómo se concretan las intenciones educativas, desde la definición más genérica correspondiente al diseño, hasta lo que ocurre en el aula" (Asociación de Unidades Académicas de Psicología, 1999, p. 5).

El primer nivel de concreción del curriculum constituye el curriculum prescripto (Coll, 1992b, 1994). En tal sentido, está relacionado con aquellos aspectos que el diseño curricular establece explícita y declarativamente como perfil, objetivos y contenidos de las carreras:

El perfil ha sido considerado como la descripción genérica del conjunto de actividades científico profesionales que debe ser capaz de desempeñar el graduado. Los objetivos han sido definidos como el colectivo de competencias o capacidades que es necesario que los estudiantes desarrollen y/o aprendan para estar en condiciones de realizar las actividades enunciadas en el perfil. Finalmente, los núcleos de contenidos y los descriptores a ser priorizados responden al análisis del desarrollo de la disciplina, tomando particularmente en cuenta la historia de la enseñanza universitaria de la psicología en nuestros países y en el mundo. (Asociación de Unidades Académicas de Psicología, 1999, p. 4).

Desde el punto de vista de la teoría didáctica y de la teoría del curriculum constructivistas, es necesario advertir que la noción de contenidos no alude únicamente a los hechos y conceptos que deber ser aprendidos, sino también a otros dos elementos centrales; por una parte, los procedimientos, por la otra, las actitudes, valores y normas (Coll, 1992a).

El segundo nivel de concreción del curriculum consiste en "la contextualización y pormenorización" del curriculum base prescripto (Coll, 1994, p. 134). En tal sentido, está relacionado con las condiciones objetivas que determinan el contexto institucional del desarrollo de los planes de estudio, desde la infraestructura de recursos hasta la elaboración o incidencia de los factores de gestión, coordinación y evaluación curricular. 
En cuanto al tercer nivel de concreción del curriculum se relaciona con la programación e implementación del plan de estudios en las prácticas docentes cotidianas y en tal sentido "son competencia y responsabilidad de cada profesor y constituyen el referente inmediato del currículum en acción, del currículum real que van a experimentar los alumnos y alumnas" (Coll, 1994, p. 134). Para la escuela elemental se lo suele denominar el curriculum en el aula, pero para el nivel superior incluye todas las prácticas de laboratorios, pasantías, practicum e internados, etc.

En relación con la enseñanza de la psicología en Argentina, y partiendo de la perspectiva teórica mencionada, el Informe diagnóstico de AUAPsi, señalaba para el primer nivel de concreción:

El último período de ruptura institucional implicó una pérdida de las tradiciones académicas, una detención del desarrollo curricular, una interrupción de la formación docente y una paralización de la investigación.

La mayoría de los planes tiene más de diez años de antigüedad.

Casi todos los planes fueron diseñados por personas no formadas en diseño curricular.

En general, no se consideraron otros modelos curriculares más allá de los nacionales.

Hubo una prevalencia de lo profesional en detrimento de lo disciplinar.

Las normativa para el cursado de las asignaturas se presentan en general como muy laxas.

Las correlatividades suelen ser poco coherentes en la secuenciación de los contenidos de las asignaturas.

Los perfiles [del plan de estudio] tienen un sesgo clínico.

Los objetivos [del plan de estudio] prácticamente no consideran el desarrollo de capacidades lingüísticas y de relaciones interpersonales, lo cual plantea una cierta incoherencia con lo establecido en los perfiles.

Los objetivos apenas consideran el desarrollo de capacidades ético-axiológico-deontológicas.

El recorte de los contenidos mínimos de las asignaturas no alcanza a cubrir las exigencias de los perfiles ni las capacidades de los objetivos.

Los procesos psicológicos básicos y las metodologías de investigación en psicología ocupan un lugar relativamente secundario en ese recorte.
Los planes dan muy poca importancia a la evaluación de alumnos y docentes, y apenas se refieren a su propia evaluación" (Asociación de Unidades Académicas de Psicología, 1998, p. 12, 15 у 21).

En el segundo nivel de concreción, en relación con la situación de los docentes y los alumnos se señalaba lo siguiente:

Las unidades académicas carecen de datos confiables y sistemáticos sobre la cantidad y las características del alumnado.

La matrícula de la mayoría de las carreras ha sido masiva en los últimos años y continúa en aumento.

Bajas dedicaciones de los docentes.

Elevado número de docentes ad-honorem.

Predominio excesivo de auxiliares docentes.

Mayoría de docentes no concursados (en particular, los auxiliares y JTP).

Elevada cantidad de alumnos por docente.

Escasez de titulaciones de posgrado.

Baja proporción de docentes categorizados dentro del Programa de Incentivos a los Docentes Investigadores.

Muy bajo número de docentes habilitados a nivel nacional para dirigir proyectos de investigación (categorizados como investigadores "A" o "B") (Asociación de Unidades Académicas de Psicología, 1998, p. 23 y 33).

También en el segundo nivel de concreción pero en relación con la infraestructura y los recursos disponibles, AUAPsi destacaba:

Insuficiencia de los presupuestos, que en su mayor parte se emplean en sueldos docentes y no docentes.

Escasa inversión en otras partidas.

Insuficiencia de espacios e infraestructura para actividades teóricas y prácticas al interior de las unidades académicas.

Insuficiencia de ámbitos institucionales y comunitarios para la realización de prácticas externas de diferente filiación.

Ausencia del material didáctico y el equipamiento mínimo necesario para la enseñanza.

El acervo bibliográfico es poco actualizado, muy limitado e insuficiente en cantidad con relación a las necesidades de los usuarios.

Es muy baja la disponibilidad de colecciones de revistas completas y actualizadas. 
Pocas unidades académicas disponen de bases de datos, cuyo uso es poco frecuente.

Los sistemas informatizados existentes para el tratamiento y gestión de la información son poco eficientes y rudimentarios" (Asociación de Unidades Académicas de Psicología, 1998, p. 40).

Por último, en el tercer nivel de concreción, en lo concerniente con el funcionamiento real de los planes de estudio, tanto en lo relativo a las prácticas de aula, como el desempeño de los estudiantes o el grado de satisfacción de los actores involucrados, el estudio de AUAPsi destacaba:

Falta de reconocimiento y legitimación de espacios de gestión y evaluación curricular.

Carencia de instancias de seguimiento.

En el nivel de los programas y en el de las prácticas de aula se produce una acentuación de la perspectiva clínica ya esbozada en los perfiles.

En el recorte de contenidos de los programas se consolidan los desequilibrios detectados en los contenidos mínimos de los planes.

En el abordaje de los contenidos se detecta un predominio de la teoría psicoanalítica.

No existen estudios sistemáticos ni se dispone de un instrumento consensuado para el análisis de las prácticas de aula.

Discrepancia de más de dos años entre la duración media prevista para las carreras en los planes de estudios y su duración real media (5,4 años y 7,5 años, respectivamente).

No existen estudios sistemáticos relativos al grado de satisfacción de los actores involucrados.

No existen estudios relativos a la valoración de instancias externas vinculadas con las Unidades Académicas donde se imparten los planes de estudios" (Asociación de Unidades Académicas de Psicología, 1998, pág. 44 y 46).

Las falencias observadas en el Informe de AUAPsi, no tenían el objetivo de invalidar las calificaciones académicas obtenidas por los graduados en psicología en Argentina y Uruguay en su conjunto. Más vale, apuntaban a identificar aquellos aspectos que se consideraba que resultaba posible corregir desde los fines de una universidad pública, verdaderamente pluralista y democrática en lo político y rigurosa y abierta a las innovaciones en relación con lo académico.
En esa dirección, la segunda etapa del Programa de Formación en Especialistas en Innovación Curricular, consensuó un conjunto de recomendaciones destinadas al mejoramiento de la formación de grado en las carreras de psicología en universidades nacionales en ambos países.

Entre las mismas, y en relación con el primer nivel de concreción del curriculum, se destacaba la necesidad de revisar la coherencia interna existente entre lo enunciado en el perfil, en los objetivos y los contenidos de los planes de estudio. Asimismo, a efectos de garantizar una "formación plurivalente", superadora del perfil marcadamente profesionalista se consideraba necesario:

Desarrollar una formación básica más sólida. Dar apertura a áreas de vacancia.

Proporcionar una adecuada formación en investigación.

Profundizar las diferentes prácticas inherentes a la disciplina.

Proponer diseños curriculares más flexibles, que posibiliten una proporción óptima de materias obligatorias y electivas.

Organizar el plan de estudios en ciclos y áreas debidamente coordinados.

Precisar y diversificar los requisitos finales para la obtención del título.

Reducir la duración real de los planes de estudio conforme a los requerimientos actuales del mundo académico, en favor de una adecuada articulación de los estudios de grado y posgrado (Asociación de Unidades Académicas de Psicología, 1999, p. 5).

En relación con el segundo nivel de concreción se subrayaban los siguientes aspectos:

Mejorar ostensiblemente la infraestructura de recursos humanos y materiales, para posibilitar una gestión adecuada de las carreras en los diferentes niveles de concreción del currículum.

Contar con recursos relativos al control de gestión docente curricular.

Legitimar las instancias que lo hacen posible.

Elaborar criterios de distribución de puntos y/o cargos docentes.

Fortalecer el trabajo inter y transdisciplinario.

Desarrollar las carreras de posgrado.

Articular las carreras de grado y posgrado para definir sus alcances. 
Disponer en el mediano plazo de una masa crítica de docentes con titulaciones de posgrado.

Tematizar, discutir y elaborar normativas que regulen el ingreso de los alumnos y el tránsito ulterior en la carrera para optimizar la formación (Asociación de Unidades Académicas de Psicología, 1999, p. 5-6).

Y en relación con en el tercer nivel de concreción el estudio de AUAPsi destacaba:

Necesidad de evaluación de los planes y programas de las asignaturas y su implementación en el aula, con el objetivo de que resulten apropiadamente articulados.

Necesidad de capacitación docente continua para optimizar las prácticas docentes.

Necesidad de tipificación adecuada de las asignaturas para una distribución equitativa de recursos (Asociación de Unidades Académicas de Psicología, 1999, p. 6).

Las recomendaciones generadas por AUAPsi, ponían el acento en las complejidades y diferentes niveles de concreción del currículum. En tal sentido, el consenso alcanzado en AUAPsi evidenciaba la toma de conciencia de que el mejoramiento de la enseñanza en las carreras de psicología, desde el ingreso a las mismas hasta la formación sistemática de posgrado, no podría resolverse a través de una simple modificación del currículum prescripto.

Al contrario, en numerosas oportunidades, los cambios curriculares se limitan a meras modificaciones en la denominación de materias o en los contenidos de los mismos, que impactan escasamente en el segundo y tercer nivel de concreción del curriculum, los cuales están sostenidos por un conjunto de condiciones objetivas cristalizadas $\mathrm{y}$ por un conjunto de tradiciones fuertemente arraigadas en las respectivas comunidades académicas.

Por otro lado, otros dos factores inciden positivamente para un mejoramiento de las condiciones de la enseñanza curricular de la psicología.

En primer lugar, el regreso al país de jóvenes graduados que obtuvieron sus posgrados en el exterior, los cuales, aun con todas las dificultades para una reinserción laboral efectiva y duradera, operan como un factor dinamizador de la estructura académica.

En segundo lugar, el papel del Mercosur y el horizonte de la libre circulación de profesionales entre los países miembros, constituye un factor poderosísimo de modificación de las tradiciones académicas locales.

Un primer paso en la dirección de la libre circulación de profesionales ha sido el ya mencionado "Protocolo de Admisión de Títulos y Grados Universitarios para el Ejercicio de Actividades Académicas en los Países del Mercosur", suscripto en 1997 (Mercosur, 1997).

De acuerdo con lo establecido por dicho Protocolo, los "Estados Partes, a través de sus organismos competentes, admitirán, al solo efecto del ejercicio de actividades académicas, los títulos de grado y postrado, conferidos por las siguientes instituciones debidamente reconocidas: Universidades, en Paraguay, Instituciones de Educación Superior, en Brasil, Instituciones Universitarias, en Argentina y Uruguay" (Mercosur, 1997, p. 2).

La limitación al ámbito académico es explícita y está ratificada en el artículo cuarto del protocolo que señala que "La admisión que se otorgue en virtud de lo establecido en el Articulo Primero, no conferirá, de por sí, derecho a otro ejercicio profesional que no sea el académico" (Mercosur, 1997, p. 2).

Aun con esas limitaciones, hay que destacar que el Plan de Acción del Sector Educativo del Mercosur (SEM) para el período 2001-2005, contempla en el área de la educación superior, tres grandes cuestiones: acreditación, movilidad y cooperación interinstitucional.

Según el primero, acreditación, se señala que un "un sistema de acreditación de carreras como mecanismo de reconocimiento de títulos de grado facilitará la movilidad en la región, estimulará los procesos de evaluación con el fin de elevar la calidad educativa y favorecerá la comparabilidad de los procesos de formación en términos de calidad académica" (Mercosur. Sector Mercosur Educativo, 2001, p. 12).

En función del segundo bloque temático, movilidad, se señala que la "constitución de un espacio común regional en la educación superior tiene uno de sus pilares en el desarrollo de programas de movilidad. Este programa abarcará proyectos y acciones de gestión académica e institucional, movilidad estudiantil, sistema de transferencia de créditos e intercambio de docentes e investigadores" (Mercosur. Sector Mercosur Educativo, 2001, p. 12).

Aun cuando los procesos de acreditación son novedosos en la región, y su implementación es despareja en los distintos países integrantes del Mercosur, eso no ha impedido el hallazgo de los primeros consensos en relación con la formación universitaria del psicólogo. 
Así, en virtud del "Protocolo de Acuerdo Marco de los Principios para la Formación de Psicólogos en los Países del Mercosur y Países Asociados", las instituciones profesionales de psicología de la región acuerdan una serie de principios básicos para la formación de psicólogos, que "deberá regir los criterios para la formación de grado del psicólogo en los países de la Región" (Comité Coordinador de Psicólogos del Mercosur y Países Asociados, 1998, p. 15).

Los criterios, sumamente generales, incluyen algunas aspiraciones que han sido constantes en la formación del psicólogo en la región como la preservación de la identidad del psicólogo, la garantía de una formación comprometida con las problemáticas sociales y la garantía de una formación generalista al nivel del grado. Por otro lado, el Protocolo enfatiza la necesidad de una formación pluralista en lo teórico y metodológico y avanza en la necesidad de "una formación básica común para el reconocimiento en todos los países de la región de un psicólogo" (Comité Coordinador de Psicólogos del Mercosur y Países Asociados, 1998, p. 16), que deberá incluir los siguientes contenidos:

Procesos psicológicos cognitivos,
motivacionales, de aprendizaje, afectivo-
emocionales, percepción, atención,
interaccionales, del desarrollo, de
personalidad, psicopatológicos.
Historia, teorías y sistemas psicológicos.
Formación en investigación psicológica.
Evaluación y diagnóstico psicológico.
Epistemología de la psicología.
Psicologías aplicadas (tradicionales y
emergentes). (Comité Coordinador de
Psicólogos del Mercosur y Países Asociados,
1998, p. 16).

¿Cuál es el alcance de ese tipo de protocolos? Por una parte, no pueden considerarse más que como criterios demasiado indeterminados que requieren de un largo proceso hasta llegar a volverse determinados y bien específicos. Por la otra, y a un mismo tiempo, constituyen indicadores claros de una vocación de acuerdo sostenida por los propios actores del campo de la psicología, con la mayor independencia y autonomía en relación con los poderes públicos.

Pero aun cuando la integración en el Mercosur aparezca como uno de los factores capaces de dinamizar la formación universitaria del psicólogo en Argentina, y aun considerando un trasfondo común en la historia cultural, política, económica y social de los países de la región, no pueden desconocerse algunos rasgos diferenciales en relación con la situación de la enseñanza universitaria de la psicología.

En relación con la acreditación de los programas universitarios en psicología, en Chile, desde un lustro atrás, el Comité Técnico de Psicología convocado por la Comisión Nacional de Acreditación de Pre-grado (CNAP), consensuó unos criterios para la evaluación de carreras de psicología (Comisión Nacional de Acreditación de Pre-grado -CNAP-. Gobierno de Chile. Ministerio de Educación. Comité Técnico de Psicología, 1998).

En Argentina, en cambio, recién en el mes de junio de 2002 se elaboró un Informe para la evaluación de carreras de posgrado. Dicho Informe ha merecido varias críticas, debido a que de los siete expertos convocados para su elaboración, solamente tres de ellos eran graduados en psicología (Comisión Nacional de Evaluación y Acreditación Universitaria CONEAU-. Ministerio de Educación. Argentina, 2002). Además, los siete convocados adherían al psicoanálisis, desconociendo la necesidad, subrayada en toda la bibliografía existente, de diversificar las orientaciones teóricas en la psicología argentina, fuertemente hegemonizada por el psicoanálisis durante varias décadas.

En relación con las carreras de grado, no existe todavía ningún criterio para la evaluación de las mismas, aun cuando en el mes de agosto de 2003, la Comisión de Asuntos Académicos del Consejo de Universidades, conformado por representantes de las universidades nacionales y de las universidades privadas, acordaron incorporar las carreras de psicología, entre aquellas sujetas a la regulación de sus títulos, debido a que el graduado en psicología puede comprometer el interés público (Consejo de Universidades. Comisión de Asuntos Académicos, 2003).

Y si bien todavía no se han acordado criterios, tanto en AUAPsi, en AUPri (Asociación de Unidades Académicas de Psicología de Universidad de Gestión Privada), institución que se organizó en mayo del 2003 y nuclea a las unidades académicas de psicología de universidades privadas, y en la propia Federación de Psicólogos de la República Argentina (FePRA) se viene trabajando para articular y consensuar propuestas, en especial sobre los tres aspectos que el artículo 43 de la ley de educación superior remarca como imprescindibles: la carga horaria mínima de los planes de estudio, los contenidos curriculares básicos y la intensidad de la formación práctica (República Argentina, 1995).

Para finalizar, me gustaría subrayar dos cuestiones que me parece atraviesan toda esta 
temática. La primera, que los criterios sobre una formación común y básica para todos los graduados en psicología, cualquiera sea su universidad de procedencia, constituye una aspiración legítima en función del interés público, siempre y cuando ello no asfixie la diversidad y la necesaria libertad y autonomía de las distintas universidades para vertebrar programas de formación originados por los integrantes de sus propias comunidades universitarias. Y la segunda cuestión, directamente relacionada con lo anterior, es que los procesos que conduzcan al establecimiento de criterios consensuados entre las distintas unidades académicas del país, requieren una amplia participación del conjunto de las comunidades universitarias involucradas como así también de los profesionales de la disciplina y de representantes de los sectores de la sociedad que demandan intervenciones psicológicos.

En ese sentido, deben agotarse todos los recursos para el debate fecundo y democrático y para el procesamiento de las diferencias entre todos los sectores involucrados. Ello implica, por lo menos, tres tipos de debates interrelacionados: en primer lugar, entre todos los actores comprometidos en la vida universitaria, es decir, docentes, graduados, alumnos y no-docentes; en segundo lugar, entre los psicólogos consagrados a la vida académica y los profesionales de la disciplina; y en tercer lugar, entre los miembros de la comunidad científica y profesional de la psicología, por una parte, y organismos no-gubernamentales, organizaciones de derechos humanos y sectores sociales en general, incluyendo a los más marginados de la sociedad, por la otra.

En la misma dirección, me parece poco conveniente dejar en manos únicamente de expertos este tipo de consensos. Por supuesto que creo que los expertos deben ser convocados; pero esa convocatoria debe estar enmarcada por una dirección política que sólo puede surgir de la participación verdaderamente democrática de los actores involucrados. Uno de los intelectuales progresistas más prestigiosos de nuestro tiempo, Pierre Bourdieu, señalaba que la falsa dicotomía entre scholarship y commitment, entre especialidad académica y compromiso, se podía resolver con la fórmula scholarship with commitment (Bourdieu, 2002).

\section{REFERÊNCIAS}

Albee, G. W. (2000). The Boulder's model fatal flaw. American Psychologist, 55 (2), 247-248.

Ardila, R. (1975). The first latin american conference on training in psychology. International Journal of Psychology, 7, 435-446.
Ardila, R. (Ed.) (1978). La profesión del psicólogo. México: Trillas.

Ascolani, A. (1988). Psicología en Rosario Rosario: Fundación Ross.

Asociación de Unidades Académicas de Psicología AUAPsi- (1996). Mejoramiento de la formación de grado en las carreras de psicología de seis Universidades Nacionales de la República Argentina. [Proyecto] Buenos Aires: Fondo de Mejoramiento de la Calidad Educativa (FOMEC).

Asociación de Unidades Académicas de Psicología -AUAPsiPrograma de formación de especialistas en innovación curricular en psicología (1998). Informe Diagnóstico de la Situación Actual. Buenos Aires: Mimeo.

Asociación de Unidades Académicas de Psicología -AUAPsi-. Programa de Formación de Especialistas en Innovación Curricular en Psicología (1999). Informe de la segunda etapa del programa de innovación curricular. protocolo de acuerdo: recomendaciones acerca de la formación universitaria en psicología en Argentina y Uruguay. Buenos Aires: Mimeo.

Asociación de Unidades Académicas de Psicología -AUAPsi(s/f). Actas y declaraciones ( Vol.1.) Buenos Aires: Mimeo.

Baker, D. \& Benjamin, L. (2000). The affirmation of the scientist-practitioner: a look back at Boulder. American Psychologist, 55 (2), 241-247.

Barrionuevo, M. S. \& García Marcos, F. (1975). Carrera de psicología en universidades argentinas. Psicología Médica, 2 (1), 83-110.

Bartram, D. (1996). Occupational standards and competencebased qualifications for professional applied psychologists in the UK. European Psychologist, 1 (3), 157-165.

Belar, C. (1992a). Education and training conferences in graduate education. In A. Puente, J. Matthews \& C. Brewer (Eds.), Teaching psychology in America: a history (pp. 285299). Washington: American Psychological Association.

Belar, C. (1992b). Conferences on internship and postdoctoral training. In A. Puente, J. Matthews \& C. Brewer (Eds.), Teaching psychology in America: a history (pp.301-310). Washington: American Psychological Association.

Belar, C. (2000). Scientist - practitioner \pm science + practice: Boulder is bolder. American Psychologist, 55 (2), 249-250.

Belar, C. \& Perry, N. W. (1992). National conference on scientist-practitioner education and training for the professional practice of psychology. American Psychologist, 47 (1), 71-75.

Benjamin, L. (2001). American psychology' s struggles with its curriculum: should a thousand flowers bloom? American Psychologist, 56 (9), 735-742

Blanco, A. (s/f). Proyecto para la mejora de las curricula en las universidades de América Latina: anteproyecto de psicología. Madrid: Mimeo.

Blanco, A. (1998). Requisitos y necesidades de formación para la psicología del siglo XXI. Revista de Psicología General y Aplicada, 51 (1), 149-172.

Blanco, A., Dembo, M., Di Doménico, C., Pineda, G. \& Rojo, M. (1993, julio). La formación del psicólogo para el año 2000. XXIV Congreso Interamericano de Psicología. Santiago de Chile: Mimeo. 
Bleger, J. (1962). Clase inaugural de la Cátedra de Psicoanálisis. Acta Psiquiátrica y Psicológica Argentina, 8 (1), 56-60.

Bleger, J. (1966). Psicohigiene y psicología institucional. Buenos Aires: Paidós.

Bourdieu, P. (2002). Los investigadores y el movimiento social. En P. Bourdieu, Pensamiento y acción (pp. 151156). Buenos Aires: Libros del Zorzal.

Coll, C. (1992a). Introducción. En C. Coll, J. I. Pozo, B. Sarabia \& E. Valls, Los contenidos en la reforma. enseñanza y aprendizaje de conceptos, procedimientos $y$ actitudes (pp. 9-18). Madrid: Santillana.

Coll, C. (1992b). Psicología y curriculum ( $3^{\mathrm{a}}$ reimpresión). Barcelona: Paidós Ibérica.

Coll, C. (1994). Posibilidades críticas en el desarrollo de la reforma curricular española. Substratum, 2 (5), 125-150.

Comisión Nacional de Acreditación de Pre-grado -CNAP-. Gobierno de Chile. Ministerio de Educación. Comité Técnico de Psicología (1998). Criterios para la evaluación de carreras de Psicología. Disponible en: http://www.cnap.cl/critesp/crit_esp1.html. ( Descargado el $24 / 10 / 2001$ )

Comisión Nacional de Evaluación y Acreditación Universitaria -CONEAU-. Ministerio de Educación. Argentina (2002). Criterios vinculados con la acreditación de las carreras de posgrado. Subcomisión de psicología. Documento de trabajo. Buenos Aires: Autor.

Comité Coordinador de Psicólogos del Mercosur y Países Asociados (1998). Protocolo de acuerdo marco de los principios para la formación de psicólogos en los países del mercosur y países asociados. En Conselho Federal de Psicologia, A psicologia no Mercosul (pp. 15-19). Brasilia: Autor.

Consejo de Universidades. Comisión de Asuntos Académicos (2003). Despacho de 17 de agosto de 2003 [que presta acuerdo para la inclusión del título de Psicólogo o Licenciado en Psicología en el régimen del artículo 43 de la Ley de Educación Superior]. Buenos Aires: Mimeo.

Dana, R. \& May, T. (Eds.). (1987). Internship training in professional psychology. New York: Hemisphere.

Danis, J. (1969). El psicólogo y el psicoanálisis. Revista Argentina de Psicología, 1, 75-82.

Danis, J., Bohoslavsky, R., Malfé, R., Ocampo, M. L. S. de, Berlín, M. \& Goldín, A. (1970). Mesa Redonda sobre 'Formación del psicólogo en la década del 70'. Revista Argentina de Psicología, 6, 109-121.

Di Doménico, C. (1996). Psicología y Mercosur: acerca de la armonización curricular. Acta Psiquiátrica y Psicológica de América Latina, 42 (3), 230-242,

Di Doménico, C. (1999a). Psicología y Mercosur: revisión comparativa de los acuerdos sobre formación de psicólogos. Acta Psiquiátrica y Psicológica de América Latina, 45 (1), 24-33.

Di Doménico, C. (1999b). La psicología en Argentina de cara al Mercosur. En, C. Di Doménico \& A. Vilanova (Eds). Formación de psicólogos en el Mercosur (pp. 97-104). Mar del Plata: Universidad Nacional de Mar del Plata.

Di Doménico, C. \& Vilanova, A. (1990a). Formación de psicólogos en Argentina. Universidad Nacional de Mar del
Plata. Psicólogo Argentino, 1 (3), 5-7

Di Doménico, C. \& Vilanova, A. (1990b). Acerca de la especialización en psicología: situación en nuestro país y en el mundo. Psicólogo Argentino, I (4), 13- 15.

Diez, P. (1994). Historia de la carrera de psicología de la Universidad Nacional de Mar del Plata (1966-1968). Mar del Plata: Universidad Nacional de Mar del Plata.

Ellis, H. C. (1992). Graduate education in psychology. Past, present and future. American Psychologist, 47 (4), 570-576.

European Federation of Professional Psychologists Associations (1992). Optimal standards for professional training in psychology. Bruselas: Author [Booklet № 3].

European Federation of Professional Psychologists Associations (2003). EFPA Activity Plan. 2003-2005. Disponible en

<http://www.efpa.be/Home/newpagina.htm> ( Descargado el 17/09/2003 )

Fox, R. E. \& Barclay, A. (1989). Let a thousand flowers bloom. Or, wed the garden? American Psychologist, 44 (1), 55-59.

Gentile, A. (1989). La carrera de psicólogo en Rosario y el proceso de profesionalización. Intercambios en Psicología, Psicoanálisis, Salud Mental, 1, 12-13.

Griggs, R. A., Proctor, D. L. \& Bujak-Johnson, A. (2002). The nonexistent common core. American Psychologist, 57 (6/7), 452-453.

Harari, R. (1970). El psicoanálisis y la profesionalización del psicólogo (a partir de 'El psicólogo y el psicoanálisis' de Juana Danis). Revista Argentina de Psicología, 3, 147-159.

Hayes, N. (1996). What makes a psychology graduate distinctive? European Psychologist, 1 (2), 130-134.

Klappenbach, H. (1987). Primeros cursos de psicología en la Universidad de Buenos Aires (1895 y 1896). Actualidad Psicológica, 137, 11-13.

Klappenbach, H. (1995). Antecedentes de la carrera de psicología en universidades argentinas. Acta Psiquiátrica y Psicológica de América Latina, 41 (3), 237-243.

Klappenbach, H. (1998). Historia de un problema de identidad en la psicología argentina. Revista de la Facultad de Psicología de la Universidad Nacional Mayor de San Marcos, 3 (4), 159-168.

Klappenbach, Hugo (2000a). El título profesional de psicólogo en Argentina: antecedentes históricos y situación actual. Revista Latinoamericana de Psicología, 32 (3), 419-446.

Klappenbach, H. (2000b). El psicoanálisis en los debates sobre el rol del psicólogo: Argentina, 1960-1975. Revista Universitaria de Psicoanálisis (Universidad de Buenos Aires), 2, 191-227.

Klappenbach, H. (2003). La investigación en carreras de grado de psicología en universidades nacionales de Argentina. En J. F. Villegas, P. Marassi \& J. P. Toro (Eds.), Problemas centrales para la formación académica y el entrenamiento profesional del psicòlogo en las américas (2 ed.) (Vol. 3, pp. 257-271). Santiago de Chile: Sociedad Interamericana de Psicología.

Lipsey, M. W. (1990). Core curriculum: an idea whose time is passed. In L. Brickman \& H. Ellis (Eds.), Preparing psychologists for the 21st century: Proccedings of the National Conference on Graduate Education in Psychology (pp. 114-119). Hillsdale: Erlbaum. 
Llapur, O. R. (1972). La psicología en Tucumán. Humanitas, 17 (23), 239-243.

Lloyd, M. \& Brewer, C. (1992). National conferences on undergraduate psychology. In A. Puente, J. Matthews \& C. Brewer (Eds.), Teaching Psychology in America: a history (pp. 263-284). Washington: American Psychological Association.

Lunt, I. (1994, julio). Science and practice in the education and training of psychologists. 23rd. International Congress of Applied Psychology. Madrid: Mimeo.

Lunt, I. (1998). Psychology in Europe: developments, challenges and opportunities. European Psychologist, 3 (2), 93-101.

Lunt, I. (1999). The professionalization of Psychology in Europe. European Psychologist, 4 (4), 240-247.

Lunt, I. (2002). A common framework for the training of psychologists in Europe. European Psychologist, 7 (3), 180191.

Mc Govern, T. V. (1992). Evolution of undergraduate curricula in Psychology, 1892-1992. In A. Puente, J. Matthews \& C. Brewer (Eds.), Teaching psychology in America: a history (pp. 13-38). Washington: American Psychological Association.

Mc Govern, T. V. , Furumoto, L., Halpern, D. F., Kimble, G.\& Mc Keachie, W. J. (1991). Liberal Education, Study in Depth, and the Arts and Sciences Major-Psychology. American Psychologist, 46 (6), 598-605.

Mercado Común del Sur -Mercosur - (1991). Tratado de Asunción. Montevideo: Mimeo.

Mercado Común del Sur -Mercosur - (1997). Protocolo de Admisión de Títulos y Grados Universitarios para el Ejercicio de Actividades Académicas en los Países del Mercosur. Asunción: Ministerio de Educación y Culto.

Mercado Común del Sur-Mercosur-. Reunión de Ministros de Educación (1991). Protocolo de Intenciones. Buenos Aires: Ministerio de Educación.

Mercado Común del Sur -Mercosur-. Sector Mercosur Educativo (2001). Plan de acción del SEM. 2001-2005. Montevideo: Autor.

Ministerio de Educación, Ciencia y Tecnología. Secretaría de Políticas Universitarias (2002). Anuario 1999-2000 de Estadísticas Universitarias. Buenos Aires: Autor

Monasterio, F. (1965). La psicología argentina. Revista de Psicología General y Aplicada, 20 (80), 907-922.

Newstead, S. (1994, julio). The psychology curriculum and the training of psychologists in Europa. 23rd. International Congress of Applied Psychology. Madrid: Mimeo.

Nixon, M. (1990). Professional training in psychology. Quest for international standards. American Psychologist, 45 (11), 1257-1262.

Peiró, J. M. \& Lunt, I. (2002). The context for the European framework for psychologist's training. European Psychologist, 7 (3), 169-179.

Peterson, R., Peterson, D., Abrams, J. \& Stricker, G. (1997).The national council of schools and programs of professional psychology educational model. Professional Psychology: Research and Practice, 28 (4), 373-386.
Piacente, T. (1994). La carrera de psicología en la Universidad Nacional de La Plata: investigación, grado y posgrado. En Facultad de Psicología de la Universidad de Buenos Aires, Primeras Jornadas de Investigación en Psicología (pp. 2730). Buenos Aires: Autor.

Piacente, T. (1998). Psicoanálisis y formación académica en psicología. Acta Psiquiátrica y Psicológica de América Latina, 44 (3), 278-284.

Piacente, T., Compagnucci, E., Schwartz, L. \& Talou, C. (2000). Aportes para un nuevo curriculum en psicología en la Universidad Nacional de La Plata. La Plata: Departamento de Psicología de la Universidad Nacional de La Plata.

Poortinga, Y. H. \& Lunt, I. (1997). Defining the competence of psychologists with a view to public accountability. European Psychologist, 2 (4), 293-300.

Project EuroPsyT (2001). A European framework for psychologists' training. Disponible en: <http://www.europsych.org>. (Descargado el 19/10/2001).

Pulverich, G. (1998). Basic legal conditions for psychologists in European countries. European Psychologist, 3 (2), 164166.

Raimy, V. C. (Ed.) (1950). Training in clinical pscyhology. New York: Prentice-Hall.

Red Iberoamericana para la Acreditación de la Calidad de la Educacion Superior (2003). Acta de la Asamblea Constitutiva de la Red Iberoamericana para la Acreditación de la Calidad de la Educación Superior (RIACES). Buenos Aires: CONEAU.

República Argentina (1995). Ley nacional [No 24.521]de educacióon superior. Buenos Aires: Ministerio de Cultura y Educación.

Rossi, L. (Ed.) (1997). La psicología antes de la profesión. Buenos Aires.: Eudeba.

Routh, D. K. (2000). Clinical psychology training: history of ideas and practices prior to 1946. American Psychologist, 55 (2), 236-241.

Shakow, D. (1942). The training of the clinical psychologist. Journal of Consulting Psychology, 6, 277-288.

Sheridan, E. P. , Matarazzo, J. D. \& Nelson, P. D. (1995). Accreditation of psychology's graduate professional education and training programs: an historical perspective. Professional Psychology: Research and practice, 26 (4), 386-392.

The Council of The European Communities (1989). Council Directive 89/48/EEC of 21 December 1988 on a general system for the recognition of higher-education diplomas awarded on completion of professional education and training of at least three years' duration. Official Journal, L 019, 24/01/1989, 16-23.

Tikkanen, T. (2002a). Historical EU directive proposal clearing the way for the europsychologist diploma. European Psychologist, 7 (2), 161-162.

Tikkanen, T. (2002b). European diploma in psychology: will it be strong or weak? European Psychologist, 7 (4), 312-313.

Tikkanen, T. (2003). European parliament busy with directive on professional recognition. European Psychologist, 8 (2), 123 . 
Tornimbeni, S, (1999). La carrera de psicología en la UNC, a los cuarenta años de su creación. El Psicoanálisis en el siglo, 7, 50-52.

Universidad de Buenos Aires (1957). Resolución № 528. En Resoluciones y Ordenanzas dictadas por el H. Consejo Universitario, 1957 (enero-julio), fs. s/n.

Universidad Nacional de Cuyo (1958). Ordenanza No 25. Mendoza: Universidad Nacional de Cuyo. Mimeo.

Vilanova, A. (1993). La formación de psicólogos en Iberoamérica. Acta Psiquiátrica y Psicológica de América Latina, 39 (3), 193-205.

Vilanova, A. (1994). Enseñanza de la psicología: el mundo y el país. Prensa Psicológica, 1, 36-37.

Vilanova, A. (1996). Enseñanza de la psicología: historia y problemas fundamentales. Cuadernos Argentinos de Historia de la Psicología, 2, 199-210.

Vilanova, A. (1997). Las deudas de la psicología en el Cono
Sur. Acta Psiquiátrica y Psicológica de América Latina, 43 (2), 260-263.

Vilanova, A. \& Di Doménico, C. (1999). La psicología en el Cono Sur. Datos para una historia. Mar del Plata: Universidad Nacional de Mar del Plata/Editorial Martin.

Wilpert, B. (1999). Barriers to the metamorphosis of European psychology. European Psychologist, 4 (4), 219-226.

Wilpert, B. (2002). Projecting an European diploma in psychology. European Psychologist, 7 (3), 221-224.

Recebido em 01/10/2003 Aceito em 20/11/2003

Endereço para correspondência: Hugo Klappenbach, Mitre, 1326, 5700, San Luis, Artetina. E-mail: hklappen @unsl.edu.ar 